\title{
A case-control proton magnetic resonance spectroscopy study confirms cerebellar dysfunction in benign adult familial myoclonic epilepsy
}

This article was published in the following Dove Press journal:

Neuropsychiatric Disease and Treatment

25 February 2015

Number of times this article has been viewed

\author{
Lili Long' \\ Yanmin Song' \\ Linlin Zhang ${ }^{2}$ \\ Chongyu $\mathrm{Hu}^{3}$ \\ Jian Gong ${ }^{2}$ \\ Lin $\mathrm{Xu}{ }^{\prime}$ \\ Hongyu Long' \\ Luo Zhou' \\ Yunci Zhang ${ }^{2}$ \\ Yong Zhang ${ }^{2}$ \\ Bo Xiao'
}

'Department of Neurology, Xiangya Hospital, Central South University,

Changsha, Hunan Province,

People's Republic of China; ${ }^{2}$ The

People's Hospital of Fuyang, Anhui

Province, People's Republic of

China; ${ }^{3}$ Department of Neurology,

Hunan Provincial People's Hospital,

People's Republic of China
Correspondence: Bo Xiao

Department of Neurology, Xiangya Hospital, Central South University,

Changsha, Hunan 410008, People's

Republic of China

$\mathrm{Tel}+8673184327216$

Email xiaobol962_xy@I63.com
Background: Benign adult familial myoclonic epilepsy (BAFME) is a rare form of epilepsy syndrome. The pathogenesis of BAFME remains unclear, though it seems to involve dysfunction of the cerebellum.

Objectives: The purpose of this study was to use proton magnetic resonance spectroscopy ( $\left.{ }^{1} \mathrm{H}-\mathrm{MRS}\right)$ to investigate whether neurochemical changes underlie abnormal brain function in BAFME.

Methods: Twelve BAFME patients from one family and 12 age- and sex-matched healthy controls were enrolled in this study. The ratios of NAA/Cr, NAA/Cho, Cho/Cr, and NAA/ $(\mathrm{Cr}+\mathrm{Cho})$ were analyzed.

Results: The BAFME patients exhibited a decreased N-acetylaspartate (NAA)/choline (Cho) ratio in the cerebellar cortex, whereas there were no significant differences in the NAA/creatine $(\mathrm{Cr}), \mathrm{Cho} / \mathrm{Cr}$, and $\mathrm{NAA} /(\mathrm{Cr}+\mathrm{Cho})$ ratios compared with healthy controls. There were no significant differences in ${ }^{1} \mathrm{H}-\mathrm{MRS}$ values in the frontal cortex or thalamus between the BAFME patients and controls. No correlation was detected between the NAA/Cho ratio in the cerebellar cortex and disease duration, myoclonus severity, or tremor severity.

Conclusion: Our results indicate clear cerebellar dysfunction in BAFME. ${ }^{1} \mathrm{H}-\mathrm{MRS}$ is a useful tool for the diagnosis of BAFME in combination with family history and electrophysiological examination.

Keywords: benign adult familial myoclonic epilepsy (BAFME), proton magnetic resonance spectroscopy $\left({ }^{1} \mathrm{H}-\mathrm{MRS}\right)$, cerebellum, cortical tremor, myoclonus

\section{Introduction}

Benign adult familial myoclonic epilepsy (BAFME) is a non-progressive syndrome that was first identified by Yasuda ${ }^{1}$ in 1991 and is characterized by distal subtle tremors and/or non-rhythmic myoclonus. Cortical myoclonus, tremor, and generalized tonicclonic seizure (GTCS) constitute the core symptoms of BAFME, which is significantly different from other epilepsy syndromes. BAFME patients suffer from rare attacks of GTCS, which are not usually accompanied by ataxia and dementia. To date, nearly 100 BAFME families (mainly in Japan and Europe) have been reported under several different disease names, including BAFME, familial adult myoclonic epilepsy (FAME), familial cortical tremor and epilepsy (FCTE), familial cortical myoclonic tremor with epilepsy (FCMTE), and autosomal dominant cortical myoclonus and epilepsy (ADCME). ${ }^{2-6}$ Genetic studies have revealed that BAFME presents significant genetic heterogeneity, and four disease loci (8q24, 2p11.1-q12.2, 5p15.31-p15.1, and $3 q 26.32-3 q 28$ ) have been identified. ${ }^{7-10}$ To date, however, no causative genes have been discovered. 
Neuropathological studies of BAFME patients have revealed cerebellar Purkinje cell degeneration that mimics to some extent that seen in SCA6 and ET. ${ }^{11}$ Cerebellar symptoms or cerebellar atrophy have also been reported in several BAFME pedigrees; ${ }^{12,13}$ hence, dysfunction of the cerebellum seems to be involved in the pathology of BAFME. However, electrophysiological studies of BAFME suggest that myoclonus originates in the cerebral cortex. The pathogenesis of BAFME is still unclear, although a "cerebello-thalamo-cortical loop hypothesis" has been proposed by some scholars. ${ }^{14-16}$ Therefore, we selected the frontal cortex, thalamus, and cerebellar cortex as areas of interest for a proton magnetic resonance spectroscopy ( ${ }^{1} \mathrm{H}-\mathrm{MRS}$ ) study. ${ }^{1} \mathrm{H}-\mathrm{MRS}$ is useful for determining changes in brain metabolites. MRS mainly reflects the concentration of N-acetylaspartate (NAA), creatine (Cr), and choline (Cho). A prior ${ }^{1} \mathrm{H}-\mathrm{MRS}$ study of an Italian BAFME family revealed an elevated $\mathrm{Cho} / \mathrm{Cr}$ ratio in the cerebellar cortex of affected family members. ${ }^{17}$ Here, we report an ${ }^{1} \mathrm{H}-\mathrm{MRS}$ study focusing on 12 Chinese BAFME patients from the same pedigree.

\section{Subjects and methods Subjects}

This study was approved by the ethics committee of Xiangya Hospital, Central South University (Changsha, People's Republic of China). Written informed consent was provided by each participant. Twelve BAFME patients were enrolled from a previously reported pedigree in which the possibility that the disease was linked to the known gene loci 8q24 or 2p11.1-q12.2 had been excluded. ${ }^{18}$ All of the BAFME patients were examined by electroencephalography (EEG); somatosensory evoked potentials (SEPs) and long-loop reflexes were also measured. The BAFME patients were diagnosed according to the diagnostic criteria described by Yasuda. ${ }^{1}$ The Unified Myoclonus Rating Scale (UMRS) and Fahn-Tolosa-Marin Tremor Rating Scale (FTRS) were used to assess the severity of myoclonus and tremor by two professional doctors in the Department of Neurology, Xiangya Hospital. ${ }^{19,20}$

\section{Magnetic resonance imaging (MRI) and 'H-MRS protocol}

All subjects were scanned on a 3.0-Tesla scanner (TIM Trio ${ }^{\circledR}$; Siemens Medical Solution, Erlangen, Germany) using a $\mathrm{Tx} / \mathrm{Rx}$-1H-head-coil. No hardware upgrades were performed during the study period.

Our MRI protocol consisted of the acquisition of three-dimensional (3D)-T1-weighted images (repetition time [TR]/echo time [TE], 1,900/3.41 ms; excitation, one time; field of view, $256 \times 256 \mathrm{~mm}$; matrix size, $256 \times 256$; slice thickness, $1 \mathrm{~mm}$ ); T2-weighted images (TR/TE, 4,000/113 ms; excitation, one time; field of view, $240 \times 240 \mathrm{~mm}$; matrix size, $320 \times 320$; slice thickness, $5 \mathrm{~mm}$ ); and T1-weighted fluid attenuated inversion recovery (TR/TE, 7,000/91 ms; field of view, $240 \times 240 \mathrm{~mm}$; matrix size, $256 \times 256$; slice thickness, $5 \mathrm{~mm}$ ). Point-resolved spectroscopy sequences were used for the MRS acquisitions with the following parameters: TR/TE, 2,000/135 ms; 128 averages, time acquisition, $257 \mathrm{~s}$. 3D-T1-weighted brain images were obtained in three orthogonal planes (sagittal, coronal, and transverse) to permit the localization of the volumes of interest (VOIs). VOIs were set according to previously used measurements combined with manual adjustments. ${ }^{17,21}$ Taking into account that all subjects were right-handed and that longer scanning times can make subjects uncomfortable, we only used the left sides of VOIs for spectrum detection. As shown in Figure 1, VOIs were individualized according to the anatomies of different subjects and located in the left frontal cortex $(18 \times 18 \times$ $20 \mathrm{~mm})$, left thalamus $(18 \times 18 \times 20 \mathrm{~mm})$, and left cerebellar cortex $(20 \times 20 \times 20 \mathrm{~mm})$. The raw data were transferred to an off-line Leonardo Workstation (Leonardo; Siemens Medical Solution). Post-processing was then performed using the built-in Siemens spectroscopy software installed on the Leonardo Workstation. We determined peak areas by integrating the corresponding signals from NAA at $2.02 \mathrm{ppm}, \mathrm{Cr}$ at $3.02 \mathrm{ppm}$, and Cho at $3.22 \mathrm{ppm}$ (Figure 2). Peak-amplitude measurement was performed independently by two skilled operators who were blinded to the subject data. The NAA/Cr, NAA/Cho, Cho/Cr, and $\mathrm{NAA} /(\mathrm{Cr}+\mathrm{Cho})$ ratios were analyzed.

\section{Statistical analysis}

Statistical analyses were performed with SPSS Statistics 19.0 software (IBM Corporation, Armonk, NY, USA). We used the Mann-Whitney $U$-test to assess differences in MRS values between BAFME patients and healthy controls. Correlations between ${ }^{1} \mathrm{H}-\mathrm{MRS}$ ratios and other parametric variables (eg, disease duration, myoclonus and tremor severity) were analyzed with Spearman's rho. The level of significance was set at $P<0.05$.

\section{Results \\ Clinical characteristics of BAFME patients and controls}

The clinical features of the patients are summarized in Table 1. A total of 12 BAFME patients (seven males and five females) were enrolled in our study. The mean age of 

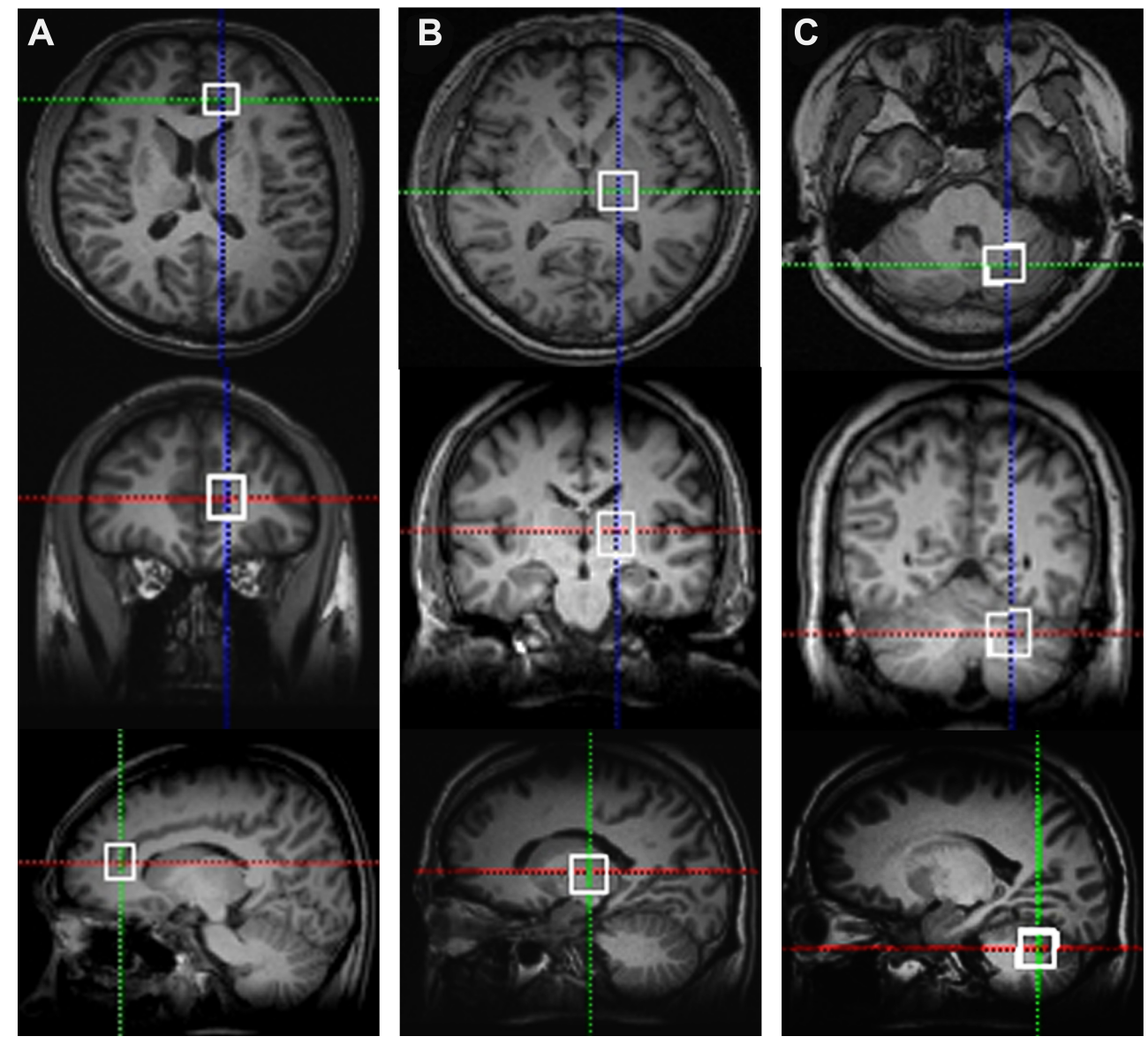

Figure I Typical spectroscopic localization of VOls from a single subject.

Notes: Transverse, coronal, and sagittal slices are shown from top to bottom. VOls: (A) left frontal cortex $(18 \times 18 \times 20 \mathrm{~mm}),(\mathbf{B})$ left thalamus $(I 8 \times 18 \times 20 \mathrm{~mm})$, and $(\mathbf{C})$ left cerebellar cortex $(20 \times 20 \times 20 \mathrm{~mm})$

Abbreviation: VOls, volumes of interest.

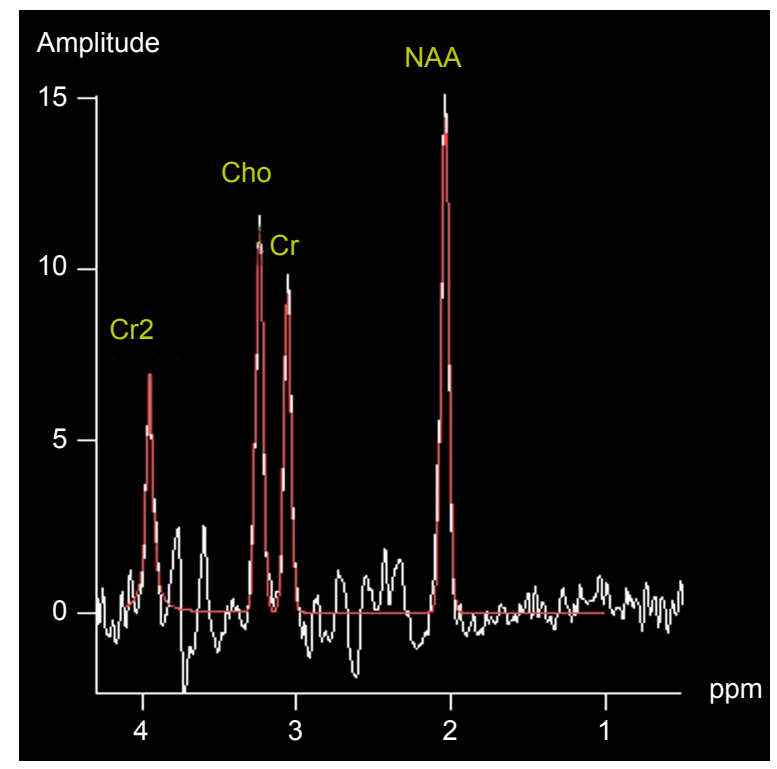

Figure 2 Sample of chemical shift imaging spectra from a subject.

Notes: Peak NAA, Cr, and Cho were determined at 2.02, 3.02, 3.22 ppm, respectively.

Abbreviations: Cho, choline; Cr, creatine; NAA, N-acetylaspartate. the patients was $42.75 \pm 11.94$ years (range: $18-58$ years). The mean onset age was $30.67 \pm 9.36$ years (range: 16-44 years), and the mean disease duration was 11.25 \pm 5.43 years (range: $2-22$ years). All of the subjects exhibited cortical myoclonus and tremors in the upper limbs. GTCS occurred in five subjects, while one patient (ID: B02) presented with frequent episodes of GTCS. None of the patients exhibited ataxia or deficits in intelligence. EEGs, SEPs, and long-loop reflexes displayed evidence of cortical reflex myoclonus in all of the patients. One patient (ID: B10) presented with a history of cerebral infarction; her MRI showed a small infarction lesion in the left parietal lobe, which did not affect VOI measurements via MRS. The T1 and T2 images of the other eleven patients were normal. The mean UMRS score was 25.25 \pm 19.08 (range: $8-76$ ), and the mean FTRS score was $20.67 \pm 10.59$ (range: 2-72). Only three patients received antiepileptic treatments (valproic acid for one patient and phenobarbital for the other two patients). Twelve healthy age-matched controls 
Table I Clinical characteristics of BAFME patients and healthy controls

\begin{tabular}{|c|c|c|c|c|c|c|c|c|c|}
\hline $\begin{array}{l}\text { Patients } \\
\text { ID }\end{array}$ & $\begin{array}{l}\text { Sex/age } \\
\text { (years) }\end{array}$ & $\begin{array}{l}\text { Onset age } \\
\text { (years) }\end{array}$ & $\begin{array}{l}\text { Duration } \\
\text { (years) }\end{array}$ & GTCS & UMRS & FTRS & AED & $\begin{array}{l}\text { Controls } \\
\text { ID }\end{array}$ & $\begin{array}{l}\text { Sex/age } \\
\text { (years) }\end{array}$ \\
\hline BOI & $M / 18$ & 16 & 2 & No & 16 & 7 & No & $\mathrm{COI}$ & $M / 18$ \\
\hline B02 & $M / 5 I$ & 32 & 19 & Yes & 76 & 72 & $\mathrm{PhB}$ & $\mathrm{C} 02$ & $M / 5 I$ \\
\hline B03 & $F / 33$ & 20 & 13 & Yes & 25 & 23 & No & $\mathrm{CO3}$ & $F / 31$ \\
\hline B04 & $M / 56$ & 40 & 16 & Yes & 35 & 26 & No & $\mathrm{CO4}$ & $M / 59$ \\
\hline B05 & $M / 36$ & 33 & 3 & No & 37 & 25 & No & $\mathrm{C} 05$ & $M / 32$ \\
\hline B06 & $M / 58$ & 40 & 18 & Yes & 24 & 37 & No & $\mathrm{C} 06$ & $M / 6 I$ \\
\hline B07 & $\mathrm{F} / 4 \mathrm{I}$ & 31 & 10 & No & 26 & 26 & No & $\mathrm{C} 07$ & $\mathrm{~F} / 46$ \\
\hline B08 & $M / 40$ & 34 & 6 & No & 11 & 8 & No & $\mathrm{C} 08$ & $M / 40$ \\
\hline B09 & $M / 38$ & 16 & 22 & Yes & 8 & 6 & VPA & $\mathrm{C} 09$ & $M / 39$ \\
\hline BIO & $F / 58$ & 44 & 14 & Yes & 29 & 10 & $\mathrm{PhB}$ & $\mathrm{ClO}$ & $\mathrm{F} / 6 \mathrm{I}$ \\
\hline BII & $F / 48$ & 36 & 12 & No & 8 & 6 & No & $\mathrm{CII}$ & $\mathrm{F} / 48$ \\
\hline $\mathrm{B} / 2$ & $F / 36$ & 26 & 10 & No & 8 & 2 & No & $\mathrm{Cl} 2$ & $\mathrm{~F} / 24$ \\
\hline Mean \pm SD & $42.75 \pm 11.94$ & $30.67 \pm 9.36$ & $11.25 \pm 5.43$ & 1 & $25.25 \pm 19.08$ & $20.67 \pm 10.59$ & 1 & 1 & $42.5 \pm 14.38$ \\
\hline
\end{tabular}

Abbreviations: BAFME, benign adult familial myoclonic epilepsy; AED, antiepileptic drug; F, female; FTRS, Fahn-Tolosa-Marin Tremor Rating Scale; GTCS, generalized tonic-clonic seizure; M, male; PhB, phenobarbital; SD, standard deviation; VPA, valproic acid; UMRS, Unified Myoclonus Rating Scale.

(seven males and five females; mean age: $42.5 \pm 14.38$ years, range: 18-61 years) with no history of neurological or psychiatric diseases were included.

\section{Morphological and metabolite 'H-MRS data}

The mean and standard deviation of the NAA/Cr, NAA/ Cho, $\mathrm{Cho} / \mathrm{Cr}$, and $\mathrm{NAA} /(\mathrm{Cr}+\mathrm{Cho})$ ratios in the BAFME patients and controls are shown in Table 2. The NAA/Cho ratio was significantly decreased in the cerebellar cortex of the BAFME patients compared with the healthy controls. The $P$-value from the Mann-Whitney $U$-test was 0.026 . A slightly increased $\mathrm{Cho} / \mathrm{Cr}$ ratio was found in the cerebellum of BAFME patients compared with the controls, but this difference was not statistically significant $(P=0.083)$. The mildly decreased NAA $/(\mathrm{Cr}+\mathrm{Cho})$ ratio in the cerebellum of BAFME patients was also not found to be statistically significant $(P=0.094)$. No significant differences were found in the ${ }^{1} \mathrm{H}$-MRS values in the frontal cortex and thalamus between the BAFME patients and controls.

\section{Correlations between 'H-MRS and clinical variables}

Among the BAFME patients, Spearman's rho analysis indicated no correlation between the NAA/Cho ratios in the cerebellar cortex and disease duration $(P=0.075)$, myoclonus severity (based on UMRS scores) $(P=0.463)$, or tremor severity (based on FTRS scores) $(P=0.475)$ (Table 3).

Table 2 'H-MRS data in BAFME patients and healthy controls

\begin{tabular}{|c|c|c|c|}
\hline \multirow[t]{2}{*}{ 'H-MRS ratios of VOls } & \multicolumn{2}{|c|}{ 'H-MRS ratios (Mean \pm SD) } & \multirow{2}{*}{$\begin{array}{l}\text { Mann-Whitney U-test } \\
\text { P-value }\end{array}$} \\
\hline & Patients $(n=\mid 2)$ & Controls $(n=\mid 2)$ & \\
\hline \multicolumn{4}{|l|}{ Frontal cortex } \\
\hline $\mathrm{NAA} / \mathrm{Cr}$ & $1.421 \pm 0.195$ & $1.400 \pm 0.246$ & 1.000 \\
\hline NAA/Cho & $1.215 \pm 0.139$ & $1.267 \pm 0.249$ & 0.435 \\
\hline $\mathrm{Cho} / \mathrm{Cr}$ & $1.175 \pm 0.171$ & $1.115 \pm 0.163$ & 0.817 \\
\hline $\mathrm{NAA} /(\mathrm{Cr}+\mathrm{Cho})$ & $0.642 \pm 0.074$ & $0.658 \pm 0.114$ & 0.582 \\
\hline \multicolumn{4}{|l|}{ Thalamus } \\
\hline $\mathrm{NAA} / \mathrm{Cr}$ & $1.82 \mathrm{I} \pm 0.263$ & $\mathrm{I} .82 \mathrm{I} \pm 0.283$ & 0.862 \\
\hline NAA/Cho & $1.911 \pm 0.180$ & $1.909 \pm 0.396$ & 0.624 \\
\hline $\mathrm{Cho} / \mathrm{Cr}$ & $0.954 \pm 0.127$ & $0.988 \pm 0.219$ & 0.340 \\
\hline $\mathrm{NAA} /(\mathrm{Cr}+\mathrm{Cho})$ & $0.933 \pm 0.097$ & $0.910 \pm 0.188$ & 0.977 \\
\hline \multicolumn{4}{|l|}{ Cerebellum } \\
\hline $\mathrm{NAA} / \mathrm{Cr}$ & $1.073 \pm 0.138$ & $1.105 \pm 0.153$ & 0.386 \\
\hline NAA/Cho & $1.165 \pm 0.123$ & $1.259 \pm 0.198$ & $0.026 *$ \\
\hline $\mathrm{Cho} / \mathrm{Cr}$ & $0.925 \pm 0.109$ & $0.885 \pm 0.105$ & 0.083 \\
\hline $\mathrm{NAA} /(\mathrm{Cr}+\mathrm{Cho})$ & $0.559 \pm 0.061$ & $0.640 \pm 0.121$ & 0.094 \\
\hline
\end{tabular}

Note: $* P<0.05$ versus controls.

Abbreviations: BAFME, benign adult familial myoclonic epilepsy; 'H-MRS, proton magnetic resonance spectroscopy; Cho, choline; Cr, creatine; NAA, N-acetylaspartate; $\mathrm{SD}$, standard deviation; VOls, volumes of interest. 
Table 3 Correlations between 'H-MRS and clinical variables

\begin{tabular}{llll}
\hline & Disease duration & $\begin{array}{l}\text { Myoclonus severity } \\
\text { (based on UMRS scores) }\end{array}$ & $\begin{array}{l}\text { Tremor severity } \\
\text { (based on FTRS scores) }\end{array}$ \\
\hline NAA/Cho ratio & $r=-0.533$ & $r=-0.234$ & $r=-0.228$ \\
in cerebellum cortex & $P=0.075$ & $P=0.463$ & $P=0.475$ \\
\hline
\end{tabular}

Notes: All $P>0.05$. " $r$ " indicates the correlation coefficient in Spearman's rho analysis.

Abbreviations: 'H-MRS, proton magnetic resonance spectroscopy; Cho, choline; FTRS, Fahn-Tolosa-Marin Tremor Rating Scale; NAA, N-acetylaspartate; UMRS, Unified Myoclonus Rating Scale.

\section{Discussion}

BAFME is considered an idiopathic epilepsy syndrome that features tremor, myoclonus, and rare GTCS as its core symptoms; BAFME is rare and not currently recognized by the International League Against Epilepsy. The pathogenesis of BAFME remains unknown because causative genes have yet to be identified. MRS has been widely used to detect neuronal dysfunction in patients with idiopathic epilepsy. NAA exists only in the neuronal mitochondria of the brain; therefore, the NAA peak reduction is attributed to neuronal loss or dysfunction. $\mathrm{Cr}$ is found in both neurons and glial cells and can be used as an internal reference because it is the most stable cerebral metabolite. Cho, a component of the cell membrane, is responsible for phospholipid metabolism. Therefore, the $\mathrm{NAA} / \mathrm{Cr}$ and $\mathrm{NAA} /(\mathrm{Cr}+\mathrm{Cho})$ ratios can be used as indicators of neuronal loss and astrocytosis, as well as to evaluate neuronal function. The $\mathrm{Cho} / \mathrm{Cr}$ ratio can be used as another indicator of neuronal dysfunction. ${ }^{22-24}$ Our study revealed an abnormal decrease in the cerebellar NAA/Cho ratio in BAFME patients compared with healthy controls. A slight increase in the $\mathrm{Cho} / \mathrm{Cr}$ ratio and a mild decrease in the $\mathrm{NAA} /(\mathrm{Cr}+\mathrm{Cho})$ ratio were also observed in the cerebellum cortex of BAFME patients; however, the differences were not statistically significant.

While electrophysiological studies have indicated that myoclonus originates in the cerebral cortex, several studies have also revealed cerebellar abnormalities in BAFME patients. Severe loss of Purkinje cells and abnormal Purkinje cell morphology with somal sprouting and loss of the dendritic tree have been observed in the cerebellum of BAFME patients during postmortem examinations. ${ }^{11,14}$ MR diffusion tensor imaging also revealed significantly decreased mean FA values in the cerebellum of FCMTE patients compared with controls. ${ }^{25}$ Abnormal cortical physiological functions and cerebellar pathology do appear to be coincidental, and similar situations have been found in diseases such as UnverrichtLundborg disease, Lafora body disease, and spinocerebellar ataxia type $14 .{ }^{26-28}$ These results demonstrate that the cerebellum plays an important role in cortical myoclonus or tremors. Thus, many scholars have proposed a "cerebellothalamo-cortical loop hypothesis", which states that cerebellar lesions decrease the number of inhibitory fibers projecting to the cortex, thereby leading to cortical excitability. However, cortical myoclonus is not observed in patients with spinocerebellar ataxia type 6 , despite the loss of Purkinje cells. ${ }^{29}$ This result indicates that the development of cortical myoclonus may not only be due to an abnormal cerebellum. Cerebellar dysfunction is likely to be just one of the mechanisms that cause or aggravate cortical myoclonus. We therefore selected VOIs in the prefrontal cortex, thalamus, and cerebellum in this study, but no abnormal alterations in the thalamus or frontal cortex were found. Striano found significantly increased $\mathrm{Cho} / \mathrm{Cr}$ ratios and slightly decreased $\mathrm{NAA} / \mathrm{Cr}$ ratios in the cerebellum in an Italian family with mutations in 2p11.1q12.2. These results differ somewhat from those of our study, possibly due to differences in genetic heterogeneity and race (in our family, the possibility of mutations associated with 2p11.1-q12.2 was ruled out). However, the decreased NAA/ Cho and increased $\mathrm{Cho} / \mathrm{Cr}$ ratios in the cerebellum of BAFME patients also reflects neuron loss and secondary astrocytosis in the cerebellum. In addition, we did not identify any obvious neural metabolic changes in the thalamus and frontal cortex of BAFME patients. This result can partially explain why only cerebellar neuronal loss and gliosis were observed in the autopsies of BAFME patients. Our results further verified the involvement of "cerebello-thalamo-cortical loop" dysfunction in the pathogenesis of BAFME. The myoclonic tremor in BAFME patients may be attributed mainly to electrophysiological changes of the cerebral cortex, which are caused by pathological changes or functional damage in the cerebellum instead of the cerebral cortex itself.

In our study, we found no correlation between ${ }^{1} \mathrm{H}-\mathrm{MRS}$ changes in the cerebellum and disease duration, severity of tremors, and severity of myoclonus. However, a notable decrease in the cerebellar NAA/Cho ratio can be observed in younger patients (for example ID: B01) with mild symptoms. While genetic diagnosis cannot be carried out, MRS may aid in diagnosing BAFME before disease onset.

This study does carry some limitations. First, we used a single-voxel ${ }^{1} \mathrm{H}-\mathrm{MRS}$ protocol, which provides limited information when focusing only on the selected VOIs. Second, ${ }^{1} \mathrm{H}-\mathrm{MRS}$ is sensitive to metabolic changes following 
epileptic seizures. Clinical heterogeneity is obvious in this BAFME pedigree, in which one patient (ID: B02) experienced significant GTCS attacks while the other patients experienced rare or no attacks. Third, we performed only unilateral scanning on the side of the brain containing the VOIs instead of scanning both sides simultaneously. We also need to examine another BAFME family to validate our findings and study the molecular mechanisms of BAFME further.

\section{Conclusion}

In this study, we found that the NAA/Cho ratio in the cerebellum of BAFME patients was significantly decreased compared with that in healthy controls. Our results indicated that cerebellar dysfunction may be involved in the pathogenesis of BAFME. ${ }^{1} \mathrm{H}-\mathrm{MRS}$ is a useful tool with which to detect brain changes in BAFME patients and may play a supporting role in the early diagnosis of BAFME when combined with family history and electrophysiological examination. In the future, other approaches, such as positron emission tomography and structural or functional MRI, should also be used to elucidate the pathogenesis of BAFME.

\section{Acknowledgments}

This project was supported by the National Natural Science Foundation of China (Grant No 81201001). The authors thank the patients and control subjects for their participation in the study.

\section{Disclosure}

All authors report no conflicts of interest in this work.

\section{References}

1. Yasuda T. Benign adult familial myoclonic epilepsy (BAFME). Kawasaki Med J. 1991;17:1-13.

2. Striano P, Zara F, Striano S. Autosomal dominant cortical tremor, myoclonus and epilepsy: many syndromes, one phenotype. Acta Neurol Scand. 2005;111(4):211-217.

3. Uyama E, Tokunaga M, Murakami T, et al. Familial adult myoclonus epilepsy: a new phenotype of autosomal dominant myoclonic epilepsy. Ann Neurol. 1996;40:505.

4. Okuma Y, Shimo Y, Shimura H, et al. Familial cortical tremor with epilepsy: an under-recognized familial tremor. Clin Neurol Neurosurg. 1998;100(1):75-78.

5. Regragui W, Gerdelat-Mas A, Simonetta-Moreau M. Cortical tremor (FCMTE: familial cortical myoclonic tremor with epilepsy). Neurophysiol Clin. 2006;36(5-6):345-349.

6. Guerrini R, Bonanni P, Patrignani A, et al. Autosomal dominant cortical myoclonus and epilepsy (ADCME) with complex partial and generalized seizures A newly recognized epilepsy syndrome with linkage to chromosome 2p11. 1-q12. 2. Brain. 2001;124(Pt 12):2459-2475.

7. Mori S, Nakamura M, Yasuda T, Ueno S, Kaneko S, Sano A. Remapping and mutation analysis of benign adult familial myoclonic epilepsy in a Japanese pedigree. J Hum Genet. 2011;56(10):742-747.
8. Madia F, Striano P, Di Bonaventura C, et al. Benign adult familial myoclonic epilepsy (BAFME): evidence of an extended founder haplotype on chromosome 2p11.1-q12.2 in five Italian families. Neurogenetics. 2008;9(2):139-142.

9. Depienne C, Magnin E, Bouteiller D, et al. Familial cortical myoclonic tremor with epilepsy The third locus (FCMTE3) maps to 5p. Neurology. 2010;74(24):2000-2003.

10. Yeetong P, Ausavarat S, Bhidayasiri R, et al. A newly identified locus for benign adult familial myoclonic epilepsy on chromosome $3 \mathrm{q} 26$. 32-3q28. Eur J Hum Genet. 2013;21(2):225-228.

11. van Rootselaar AF, Aronica E, Jansen Steur EN, RozemullerKwakkel JM, de Vos RA, Tijssen MA. Familial cortical tremor with epilepsy and cerebellar pathological findings. Mov Disord. 2004;19(2): 213-217.

12. van Rootselaar F, Callenbach PM, Hottenga JJ, et al. A Dutch family with 'familial cortical tremor with epilepsy'. Clinical characteristics and exclusion of linkage to chromosome 8q23.3-q24.1. J Neurol. 2002; 249(7):829-834.

13. Suppa A, Berardelli A, Brancati F, et al. Clinical, neuropsychological, neurophysiologic, and genetic features of a new Italian pedigree with familial cortical myoclonic tremor with epilepsy. Epilepsia. 2009;50(5): 1284-1288.

14. Tijssen MA, Thom M, Ellison DW, et al. Cortical myoclonus and cerebellar pathology. Neurology. 2000;54(6):1350-1356.

15. Regragui W, Gerdelat-Mas A, Simonetta-Moreau M. Cortical tremor (FCMTE: familial cortical myoclonic tremor with epilepsy). Neurophysiol Clin. 2006;36(5-6):345-349.

16. van Rootselaar AF, van der Salm SM, Bour LJ, et al. Decreased cortical inhibition and yet cerebellar pathology in 'familial cortical myoclonic tremor with epilepsy'. Mov Disord. 2007;22(16):2378-2385.

17. Striano P, Caranci F, Di Benedetto R, et al. ${ }^{1} \mathrm{H}-\mathrm{MR}$ spectroscopy indicates prominent cerebellar dysfunction in benign adult familial myoclonic epilepsy. Epilepsia. 2009;50(6):1491-1497.

18. Deng FY, Gong J, Zhang YC, et al. Absence of linkage to 8q23.3-q24. 1 and 2p11. 1-q12. 2 in a new BAFME pedigree in China: Indication of a third locus for BAFME. Epilepsy Res. 2005;65(3):147-152.

19. Frucht SJ, Leurgans SE, Hallett M, Fahn S. The unified myoclonus rating scale. Adv Neurol. 2002;89:361-376.

20. Fahn S, Tolosa E, Marin C. Clinical Rating Scale for Tremor. In: Jankovic J, Tolosa E, editors. Parkinson's Disease and Movement Disorders. 2nd ed. Baltimore, MD: Williams \& Wilkins; 1993:271-280.

21. Mory SB, Li LM, Guerreiro CA, Cendes F. Thalamic dysfunction in juvenile myoclonic epilepsy: a proton MRS study. Epilepsia. 2003; 44(11):1402-1405.

22. Urenjak J, Williams SR, Gadian DG, Noble M. Proton nuclear magnetic resonance spectroscopy unambiguously identifies different neural cell types. J Neurosci. 1993;13(3):981-989.

23. Petroff OA, Errante LD, Rothman DL, Kim JH, Spencer DD. Neuronal and glial metabolite content of the epileptogenic human hippocampus. Ann Neurol. 2002;52(5):635-642.

24. Maton BM, Kuzniecky RI. Proton MRS: N-acetyl aspartate, creatine, and choline. Adv Neurol. 2000;83:253-259.

25. Buijink AW, Caan MW, Tijssen MA, et al. Decreased cerebellar fiber density in cortical myoclonic tremor but not in essential tremor. Cerebellum. 2013;12(2):199-204.

26. Shahwan A, Farrell M, Delanty N. Progressive myoclonic epilepsies: a review of genetic and therapeutic aspects. Lancet Neurol. 2005;4(4):239-248.

27. Sharifi S, Aronica E, Koelman JH, Tijssen MA, Van Rootselaar AF. Familial cortical myoclonic tremor with epilepsy and cerebellar changes: description of a new pathology case and review of the literature. Tremor Other Hyperkinet Mov (N Y). 2012;2. pii: tre-02-82-472-2.

28. Ganos C, Zittel S, Minnerop M, et al. Clinical and neurophysiological profile of four German families with spinocerebellar ataxia type 14 . Cerebellum. 2014;13(1):89-96.

29. Yang Q, Hashizume $Y$, Yoshida M, et al. Morphological Purkinje cell changes in spinocerebellar ataxia type 6. Acta Neuropathol. 2000;100(4):371-376. 


\section{Publish your work in this journal}

Neuropsychiatric Disease and Treatment is an international, peerreviewed journal of clinical therapeutics and pharmacology focusing on concise rapid reporting of clinical or pre-clinical studies on a range of neuropsychiatric and neurological disorders. This journal is indexed on PubMed Central, the 'PsycINFO' database and CAS, and is the official journal of The International Neuropsychiatric Association (INA). The manuscript management system is completely online and includes a very quick and fair peer-review system, which is all easy to use. Visit http://www.dovepress.com/testimonials.php to read real quotes from published authors.

\footnotetext{
Submit your manuscript here: http://www.dovepress.com/neuropsychiatric-disease-and-treatment-journal
} 